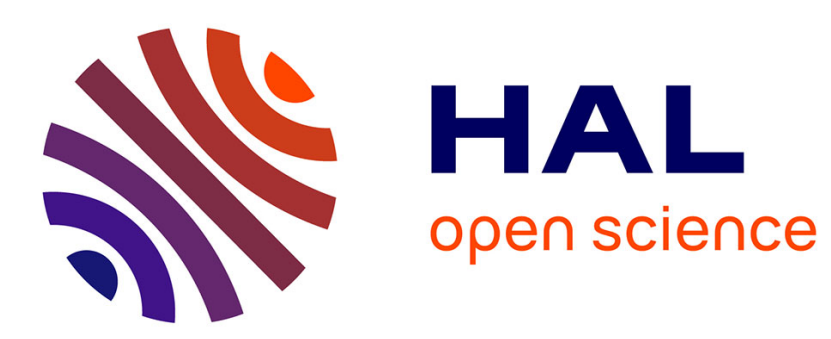

\title{
Real-time synthesis of clarinet-like instruments using digital impedance models
}

Philippe Guillemain, Jean Kergomard, Thierry Voinier

\section{To cite this version:}

Philippe Guillemain, Jean Kergomard, Thierry Voinier. Real-time synthesis of clarinet-like instruments using digital impedance models. Journal of the Acoustical Society of America, 2005, 118, pp.483-494. 10.1121/1.1937507 . hal-00009254

\section{HAL Id: hal-00009254 \\ https://hal.science/hal-00009254}

Submitted on 12 Jun 2020

HAL is a multi-disciplinary open access archive for the deposit and dissemination of scientific research documents, whether they are published or not. The documents may come from teaching and research institutions in France or abroad, or from public or private research centers.
L'archive ouverte pluridisciplinaire HAL, est destinée au dépôt et à la diffusion de documents scientifiques de niveau recherche, publiés ou non, émanant des établissements d'enseignement et de recherche français ou étrangers, des laboratoires publics ou privés. 


\title{
Real-time synthesis of clarinet-like instruments using digital impedance models
}

\author{
Philippe Guillemain, ${ }^{\text {a) }}$ Jean Kergomard, and Thierry Voinier \\ CNRS-Laboratoire de Mécanique et d'Acoustique, 31 Chemin Joseph Aiguier, 13402 Marseille \\ Cedex 20, France
}

\begin{abstract}
A real-time synthesis model of wind instruments sounds, based upon a classical physical model, is presented. The physical model describes the nonlinear coupling between the resonator and the excitor through the Bernoulli equation. While most synthesis methods use wave variables and their sampled equivalent in order to describe the resonator of the instrument, the synthesis model presented here uses sampled versions of the physical variables all along the synthesis process, and hence constitutes a straightforward digital transposition of each part of the physical model. Moreover, the resolution scheme of the problem (i.e., the synthesis algorithm) is explicit and all the parameters of the algorithm are expressed analytically as functions of the physical and the control parameters.
\end{abstract}

\section{INTRODUCTION}

The calculation of the self-sustained oscillations of single-reed instruments such as the clarinet was proposed for the first time by Schumacher, ${ }^{1}$ using a time domain discretization of the equations. This work, which does not attempt a real-time implementation, has later been widely developed (see, e.g., Gazengel et $_{\text {al. }}{ }^{2}$ or Ducasse ${ }^{3}$ ). Numerical methods, such as the harmonic balance, have been developed by Gilbert ${ }^{4}$ in order to study the periodic solutions. In addition, in order to perform the calculations in real-time, several methods yielding a time domain formulation of the waves in the resonator have been developed. We can mention the wellknown digital waveguide method (see, e.g., Smith $^{5}$ or Välimäki ${ }^{6}$ ) and wave digital modeling used by van Walstijn, ${ }^{7}$ which consider the incoming and outgoing waves within each section of a bore and their scattering at the junctions between bores of different sections. We can also mention the calculation of the reflection function from impedance measurements using inverse Fourier transform, which has been used for the synthesis of the trumpet by Vergez. ${ }^{8}$ Like the Schumacher method, since these methods consider the incoming and outgoing waves, the problem then lays in the formulation of the nonlinearity which, whatever the model, can only be expressed physically in terms of acoustic pressure and flow at the mouthpiece of the instrument.

In this paper, a real-time synthesis model using only physical variables is proposed. It first consists in directly expressing the impedance relationship at the mouthpiece between flow and pressure, which become the input and output of the linear part of the model characterizing the bore. The nonlinear coupling (based on the steady Bernoulli flow model) between the bore and the reed (described classically as a single mass-spring damped oscillator) is then modeled in a physical way. In the case of sampled signals, thanks to a suitable discretization scheme of the reed displacement, this

${ }^{\text {a)} E l e c t r o n i c ~ m a i l: ~ g u i l l e m @ 1 m a . c n r s-m r s . f r ~}$ coupling is solved explicitly whatever the shape of the bore without iterative or table-lookup schemes such as the K-Method described by Borin. ${ }^{9}$

The paper is organized as follows. Section II presents the classical physical model in the case of a cylindrical bore (see, e.g., Kergomard et al. ${ }^{10}$ ) allowing the construction of the main elements of the synthesis model. Section III presents a preliminary step toward the synthesis algorithm in the case of a cylindrical bore, extends it to the case of a truncated conical bore, and builds the complete synthesis model. A more complete formalism for bores of other geometries and different reed models is described in Guillemain et al. ${ }^{11}$ Section IV deals with sampled variables, and presents an explicit time-domain scheme which solves the coupled system constituting the sampled physical model, its digital implementation, and its real-time control. Section V is devoted to conclusions and perspectives of this work.

\section{SIMPLIFIED PHYSICAL MODEL}

We first briefly describe the three components of the classical physical model. They are made of a linear impedance relationship between acoustic pressure and flow at the mouthpiece, a pressure driven linear oscillator modeling the first mode of vibrations of the reed, and a nonlinear characteristics coupling the flow to the pressure and the reed displacement at the mouthpiece.

\section{A. Wave equation in a cylindrical lossy bore 1. Wave number expression}

The first linear part of the physical model corresponds to the resonator of the instrument. We here consider a cylindrical bore. For such a geometry, if one assumes that the radius of the bore is large in front of the boundary layer thicknesses, the classical Kirchhoff's theory (see, e.g., Pierce ${ }^{12}$ ) leads, in the frequency domain, to the value of the complex wave number $k(\omega)$ for a plane wave: 


$$
k(\omega)^{2}=\frac{\omega^{2}}{c^{2}}\left(1-i^{3 / 2} \alpha c^{2} \omega^{-1 / 2}\right),
$$

where

$$
\alpha=2 /\left(R c^{3 / 2}\right)\left(\sqrt{l_{v}}+\left(\frac{c_{p}}{c_{v}}-1\right) \sqrt{l_{t}}\right) .
$$

$R$ is the radius of the bore: $R=7 \cdot 10^{-3}$ in the clarinet case. Typical values of the physical constants, in $\mathrm{mK} \mathrm{s}$ units, are: $c=340, l_{v}=4 \cdot 10^{-8}, l_{t}=5.6 \cdot 10^{-8}, C_{p} / C_{v}=1.4$.

For the acoustic pressure, this corresponds to the time domain differential equation (see, e.g., Polack ${ }^{13}$ ):

$$
\frac{\partial^{2} p(x, t)}{\partial x^{2}}-\frac{1}{c^{2}} \frac{\partial^{2} p(x, t)}{\partial t^{2}}-\alpha \frac{\partial^{3 / 2} p(x, t)}{\partial t^{3 / 2}}=0 .
$$

To the same order of approximation, the classical expression of $k(\omega)$, that we shall use in the following is

$$
k(\omega)=\frac{\omega}{c}-\frac{i^{3 / 2}}{2} \alpha c \omega^{1 / 2} .
$$

\section{Expression of the losses}

Let us consider a bore of infinite length, excited in $x$ $=0$ at $t=0$ by a Dirac impulse $\delta(x) \delta(t)$. At any point $x>0$, the acoustic pressure propagating from this source in the $(x$ $>0$ ) direction can be written as a continuous sum of all the waves that can propagate in the bore:

$$
p(x, t)=\int \exp (-i k(\omega) x) \exp (i \omega t) d \omega,
$$

which is the inverse Fourier transform of $\exp (-i k(\omega) x)$. The filtering of a pressure wave propagating in the $(x>0)$ direction between $x=0$ and $x=L$, including propagation delay, dispersion, and dissipation is then given by

$$
\begin{aligned}
F(\omega)= & \exp (-i k(\omega) L) \\
= & \exp \left(-\frac{1}{2} \alpha c \sqrt{\frac{\omega}{2}} L\right) \\
& \times \exp \left(-i\left(\frac{\omega}{c} L+\frac{1}{2} \alpha c \sqrt{\frac{\omega}{2}} L\right)\right) .
\end{aligned}
$$

Dissipation, present in the modulus of $F(\omega)$, and dispersion, present in the nonlinear part of the phase of $F(\omega)$ are functions of frequency, and depend both on the radius and the length of the bore.

Figure 1 shows the behavior of the modulus of $F(\omega)$ as function of frequency. This filter expresses the modifications encountered by a pressure wave when it travels from one end of the bore to the other end.

\section{Input impedance of a cylindrical bore (frequency domain)}

For a cylindrical resonator, according to the transmission line theory, the Fourier transforms $\left[P_{e}(\omega), U_{e}(\omega)\right.$ and $P_{s}(\omega)$,

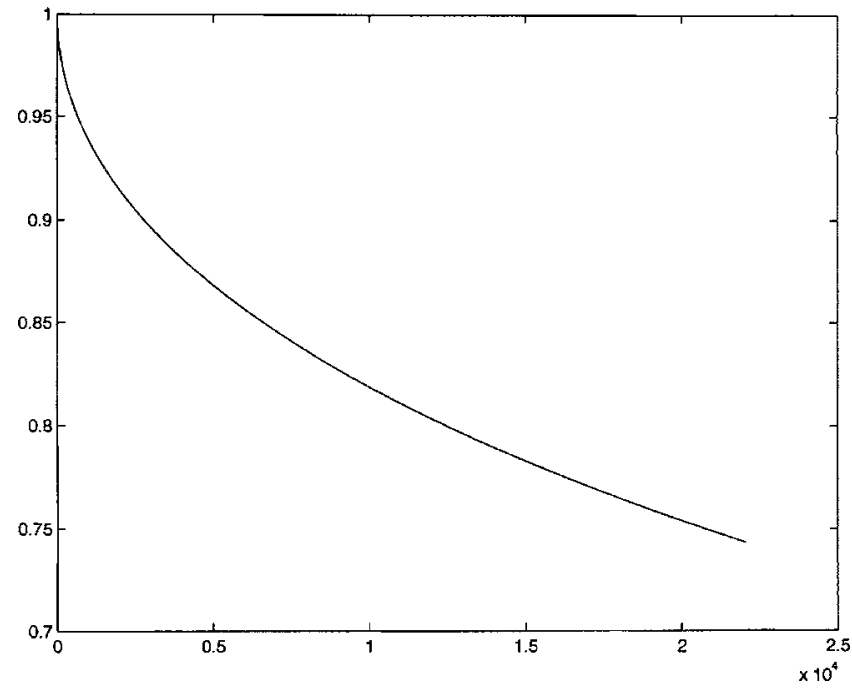

FIG. 1. Modulus of $F(\omega)$ as function of the frequency (in hertz). $L=0.5 \mathrm{~m}$, $R=7 \mathrm{~mm}$.

$\left.U_{s}(\omega)\right]$ of the dimensionless acoustic pressure and flow at the input $\left[p_{e}(t), u_{e}(t)\right]$ and at the open end $\left[p_{s}(t), u_{s}(t)\right]$ of the resonator are linked by

$$
\begin{aligned}
& P_{e}(\omega)=\cos (k(\omega) L) P_{s}(\omega)+i \sin (k(\omega) L) U_{s}(\omega), \\
& U_{e}(\omega)=i \sin (k(\omega) L) P_{s}(\omega)+\cos (k(\omega) L) U_{s}(\omega) .
\end{aligned}
$$

In these equations, the acoustic pressure $p_{e, s}$ and flow $u_{e, s}$ are normalized with respect to the physical variables $\tilde{p}_{e, s}$ and $\tilde{u}_{e, s}$ in the following way: $p_{e, s}=\tilde{p}_{e, s} / p_{M}$ and $u_{e, s}$ $=Z_{c} / p_{M} \widetilde{u}_{e, s}$, where $Z_{c}=\rho c /\left(\pi R^{2}\right)$ is the characteristic impedance of the resonator. The pressure $p_{M}$ is the static beatingreed pressure, depending on the characteristics of the reed and defined in the Sec. II B.

As a first approximation, we assume that the radius of the bore is small compared to the wavelength. In this case, the radiation losses are negligible and the radiation effect is a length correction, taken into account in $L$. Under this classical hypothesis, the output impedance $P_{s}(\omega) / U_{s}(\omega)$ vanishes and only the internal losses contained in $k(\omega)$ are taken into account. This simplification let us express the relationship between acoustic pressure and flow at the input of the resonator in the following way:

$$
P_{e}(\omega)=i \tan (k(\omega) L) U_{e}(\omega)=Z_{e}(\omega) U_{e}(\omega),
$$

where $Z_{e}(\omega)=i \tan (k(\omega) L)$ is the dimensionless input impedance of the resonator.

\section{B. Simple reed model}

We here consider a reed model based upon a classical single mode model. This model describes in a simple way the dimensionless displacement $x(t)$ of the reed with respect to its equilibrium point $x=0$ when it is submitted to a dimensionless acoustic pressure $p_{e}(t)$ : 


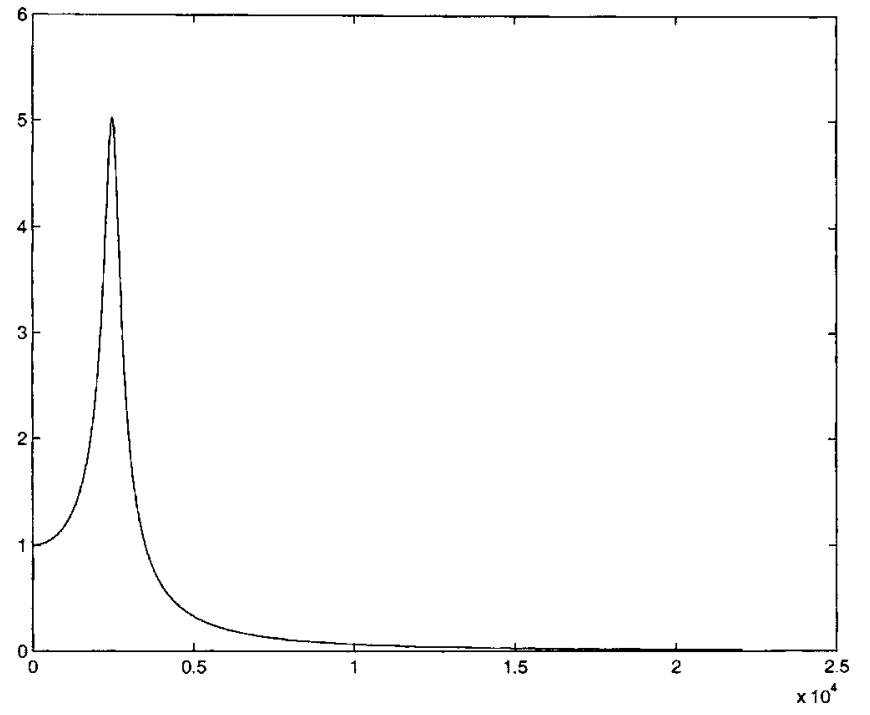

FIG. 2. Transfer function of the reed model (horizontal axis in hertz) $\left(f_{r}\right.$ $=2500, q_{r}=0.2$ ).

$$
\frac{1}{\omega_{r}^{2}} \frac{d^{2} x(t)}{d t^{2}}+\frac{q_{r}}{\omega_{r}} \frac{d x(t)}{d t}+x(t)=p_{e}(t)
$$

where $\omega_{r}=2 \pi f_{r}$ and $q_{r}$ are, respectively, the circular frequency and the quality factor of the reed. Typical values for these parameters are: $f_{r}=2500 \mathrm{~Hz}$ and $q_{r}=0.2$ in the clarinet case.

The dimensionless displacement $x$ of the reed is defined from the physical reed displacement $\tilde{y}$ by: $x=\tilde{y} / H+p_{m} / p_{M}$, where $H$ is the height of the opening between the reed and the mouthpiece and $p_{m}$ is the static (slowly varying) pressure within the mouth. The static beating-reed pressure is expressed by: $p_{M}=\mu_{r} H \omega_{r}^{2}$, where $\mu_{r}$ is the mass per unit surface of the reed.

Assuming that the initial conditions of the reed movement correspond to a displacement and a velocity equal to zero, the Fourier domain equivalent of this equation gives the transfer function of the reed:

$$
\frac{X(\omega)}{P_{e}(\omega)}=\frac{\omega_{r}^{2}}{\omega_{r}^{2}-\omega^{2}+i \omega q_{r} \omega_{r}} .
$$

The reed displacement due to an impulse pressure $p_{e}(t)$ $=\delta(t)$ is then given by

$$
x(t)=\frac{2 \omega_{r}}{\sqrt{4-q r^{2}}} \exp \left(-\frac{1}{2} \omega_{r} q_{r} t\right) \sin \left(\frac{1}{2} \sqrt{4-q r^{2}} \omega_{r} t\right) .
$$

Equation (7) shows that the response of the reed to an impulse can be considered noninstantaneous, since $x(0)=0$. Since the relation between $x(t)$ and $p_{e}(t)$ is a convolution, this property will be true for any excitation $p_{e}(t)$, and we will take advantage of this in the following.

Figures 2 and 3 show, respectively, the transfer function and the impulse response of this reed model.

\section{Nonlinear characteristics}

The dimensionless acoustic pressure $p_{e}(t)$, the dimensionless acoustic flow $u_{e}(t)$, and the dimensionless reed dis-

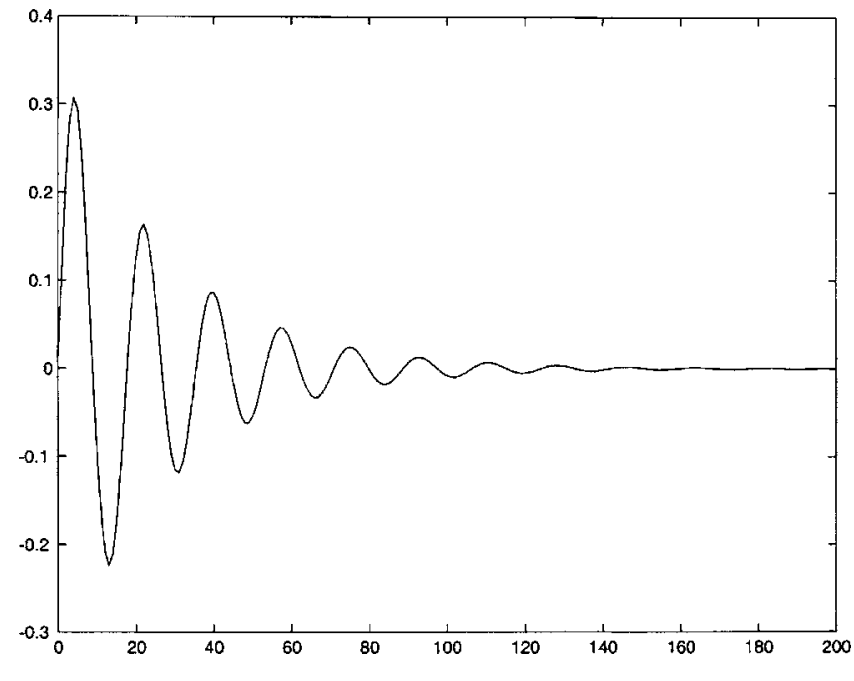

FIG. 3. Impulse response of the reed model (in samples, $f_{e}=44100$ ).

placement $x(t)$ are linked in a nonlinear way at the input of the resonator through the stationary Bernoulli equation:

$$
\begin{aligned}
u_{e}(t)= & \Theta(1-\gamma+x(t)) \zeta(1-\gamma+x(t)) \\
& \times \operatorname{sign}\left(\gamma-p_{e}(t)\right) \sqrt{\left|\gamma-p_{e}(t)\right|},
\end{aligned}
$$

where $\Theta$ is the Heaviside function, the role of which is to keep the opening of the reed positive.

This simplified nonlinear characteristics is obtained through classical hypothesis (see, e.g., Hirschberg ${ }^{14}$ and $\operatorname{Kergomard}^{15}$ ). The opening of the reed channel is proportional to the reed displacement. The acoustic flow generated by the reed (proportional to the reed velocity) and the unsteady term in the Bernoulli equation (proportional to the time derivative of the acoustic velocity) are ignored.

The parameter $\zeta$ is characteristic of the whole embouchure and is related to the maximum flow entering the instrument. It is defined by Kergomard, ${ }^{15}$ after Wilson and Beavers, ${ }^{16}$ and depends on the ratio between the reed opening and the tube cross-section area and on the reed stiffness: $\zeta=Z_{c} w H \sqrt{2 /\left(\rho p_{M}\right)}$, where $w$ is the width of the reed. Common values lay between 0.2 and 0.6 in the clarinet case. The parameter $\gamma$ is the ratio between the pressure inside the mouth of the player and the static beating-reed pressure: $\gamma$ $=p_{m} / p_{M}$. For a lossless bore and a massless reed, it evolves from $1 / 3$, which is the oscillation threshold, to $1 / 2$, which corresponds to the threshold of beating-reed. The parameters $\zeta$ and $\gamma$ constitute two important performance parameters since they, respectively, represent the way the player holds the reed and its blowing pressure inside the instrument. Though, for clarity in the notations, the $t$ variable is omitted, the playing parameters $\gamma$ and $\zeta$ are functions of time, but slowly varying compared to the other time-dependent variables.

Figure 4 represents the nonlinear characteristics of the reed for the limit case $\omega_{r}=\infty$. In this case, the displacement $x(t)$ of the reed reduces to the acoustic pressure $p_{e}(t)$ itself and the reed behaves as a single spring. 


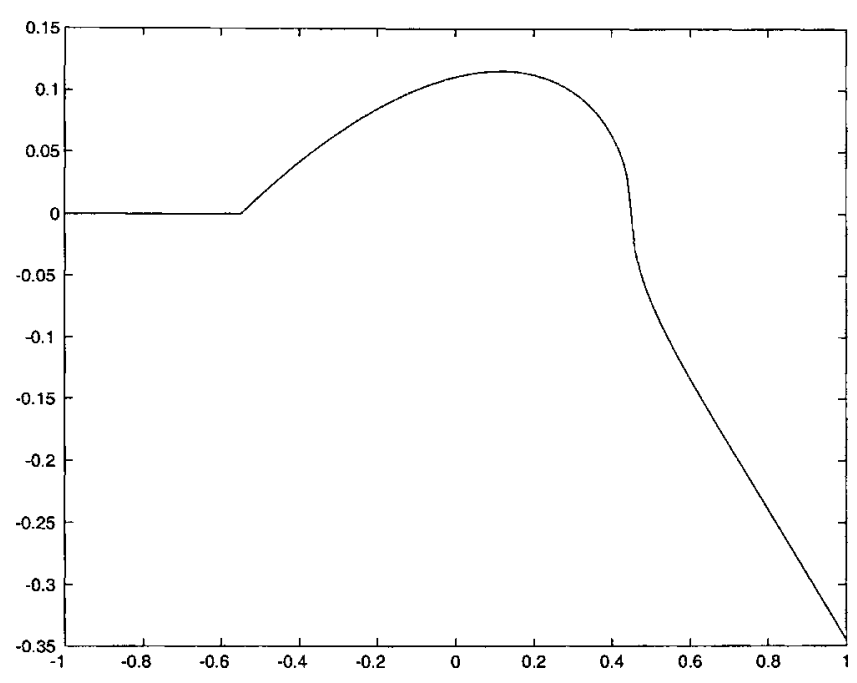

FIG. 4. Nonlinear characteristics of the reed ( $u$ as a function of $p, \zeta=0.3$, $\gamma=0.45)$.

\section{Coupling of the reed and the cylindrical resonator}

Combining the reed displacement equation, the impedance relation, and the nonlinear characteristics, the acoustic pressure at the mouthpiece level can finally be found by solving the following set of coupled equations:

$$
\begin{aligned}
\frac{1}{\omega_{r}^{2}} \frac{d^{2} x(t)}{d t^{2}}+\frac{q_{r}}{\omega_{r}} \frac{d x(t)}{d t}+x(t)=p_{e}(t), \\
P_{e}(\omega)=Z_{e}(\omega) U_{e}(\omega), \\
u_{e}(t)=\Theta(1-\gamma+x(t)) \zeta(1-\gamma+x(t)) \\
\quad \times \operatorname{sign}\left(\gamma-p_{e}(t)\right) \sqrt{\left|\gamma-p_{e}(t)\right|} .
\end{aligned}
$$

The following sections will present a way of solving these equations in the time domain for sampled variables.

\section{CONSTRUCTION OF THE SYNTHESIS MODEL FROM THE PHYSICAL MODEL}

In order to solve this system of three equations, we first propose a different formulation of the impedance relation, compatible with a time-domain implementation. We first consider the case of a cylindrical bore, and further extend it to the case of a conical bore.

\section{A. Expression of the input impedance of the resonator}

\section{Cylindrical bore}

The input impedance, denoted $\mathcal{C}(\omega)$, of a cylindrical resonator is first described as a combination of looped filters. For that purpose, the impedance $Z_{e}(\omega)$ is written as

$$
\begin{aligned}
\mathcal{C}(\omega) & =Z_{e}(\omega)=i \tan (k(\omega) L)=i \frac{\sin (k(\omega) L)}{\cos (k(\omega) L)} \\
& =\frac{\exp (i k(\omega) L)-\exp (-i k(\omega) L)}{\exp (i k(\omega) L)+\exp (-i k(\omega) L)} .
\end{aligned}
$$

This last expression can be written as follows:

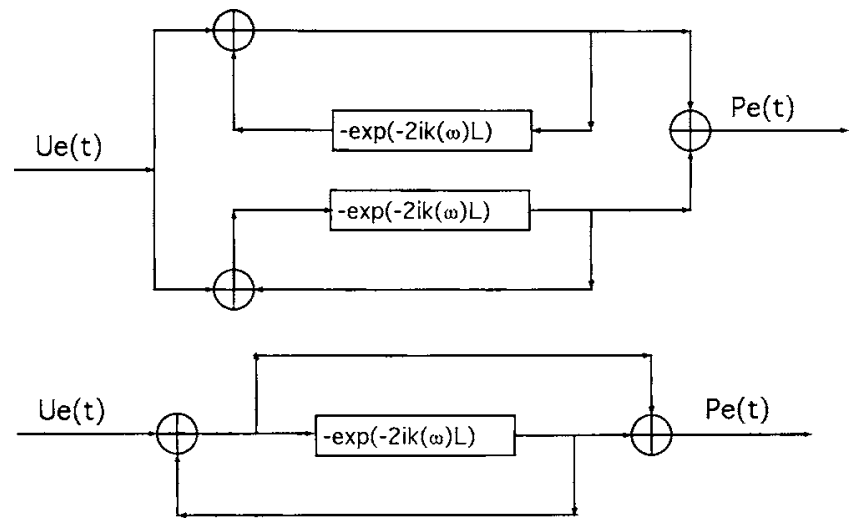

FIG. 5. Top panel: (a) Model representing the input impedance of a cylindrical resonator. Bottom panel: (b) Same model using a modified Schroeder representation.

$$
\begin{aligned}
\mathcal{C}(\omega) & =\frac{1-\exp (-2 i k(\omega) L)}{1+\exp (-2 i k(\omega) L)} \\
& =\frac{1}{1+\exp (-2 i k(\omega) L)}-\frac{\exp (-2 i k(\omega) L)}{1+\exp (-2 i k(\omega) L)} .
\end{aligned}
$$

Figure 5(a) shows the interpretation of Eq. (12) in terms of looped filters. The transfer function of this model is directly the dimensionless input impedance of a cylindrical resonator. It is made of a sum of two elementary blocks. The upper block corresponds to the first term of the second equality in Eq. (12) and the lower block corresponds to the second term.

Figure 5(b) shows that the impedance $\mathcal{C}(\omega)$ of the cylindrical bore can be related to a Schroeder ${ }^{17}$ all-pass filter, used for reverberation effects, by replacing the loop delay of the form $\exp (-i \omega D)$ by $-\exp (-2 i k(\omega) L)$ and removing the gains and their signs. This interpretation will make the digital implementation of such filters similar up to the losses contained in $\exp (-2 i k(\omega) L)$.

Figure 6 shows (top panel) the input impedance of the resonator with respect to frequency and (bottom panel) its
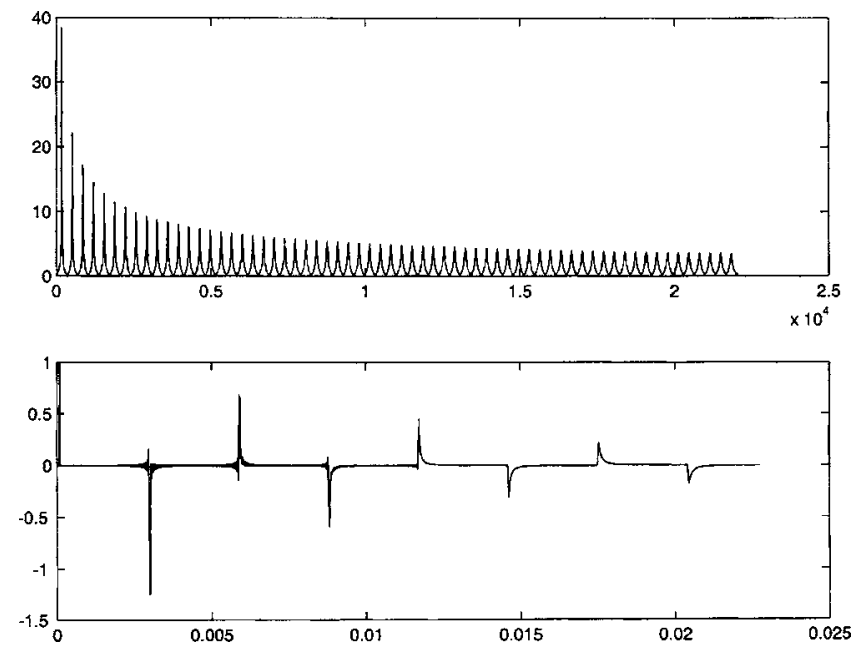

FIG. 6. Top panel: Input impedance of a cylindrical resonator ( $f$ in hertz). Bottom panel: Impulse response of a cylindrical resonator ( $t$ in seconds). 
impulse response computed through an inverse Fourier transform. Bore length: $L=0.5 \mathrm{~m}$, radius $R=7 \mathrm{~mm}$.

Similarly, we denote by $\mathcal{C}^{-1}(\omega)$ the admittance of a cylindrical bore:

$$
\mathcal{C}^{-1}(\omega)=\frac{1+\exp (-2 i k(\omega) L)}{1-\exp (-2 i k(\omega) L)} .
$$

It is worth noticing that in the case of a perfectly cylindrical bore, Eq. (12) is not the most efficient way to describe the resonator. Indeed, in this case, the description based on wave variables and used, e.g., in the digital waveguides models is more efficient.

If we decompose the acoustic pressure and flow into wave variables: $P_{e}(\omega)=P_{+}(\omega)+P_{-}(\omega)$ and $U_{e}(\omega)=P_{+}(\omega)$ $-P_{-}(\omega)$, we obtain $P_{+}(\omega)=\left(P_{e}(\omega)+U_{e}(\omega)\right) / 2$ and $P_{-}(\omega)$ $=\left(P_{e}(\omega)-U_{e}(\omega)\right) / 2$. By denoting $\mathcal{R}(\omega)$ the reflection function satisfying $P_{-}(\omega)=\mathcal{R}(\omega) P_{+}(\omega)$, it becomes

$$
\mathcal{R}(\omega)=\frac{Z_{e}(\omega)-1}{Z_{e}(\omega)+1}=-F(\omega)^{2}=-\exp (-2 i k(\omega) L) .
$$

This last equation shows that the reflection function $\mathcal{R}(\omega)$ models the back and forth propagation of the pressure waves themselves, while Eq. (12) directly models the consequence of the propagation through the introduction of the reflection function inside two loops. This constitutes a difference between the method described here and wave variables methods, though both of them are physically equivalent since they consider the bore as an acoustic waveguide.

\section{Conical bore}

In the case of a conical bore, by assuming again that the radiation impedance can be ignored and by considering the propagation of spherical waves, the input impedance, denoted $\mathcal{S}(\omega)$, is written classically as a parallel combination of a cylindrical bore and an "air" bore:

$$
\mathcal{S}(\omega)=\frac{1}{\frac{1}{i \omega \frac{x_{e}}{c}}+\frac{1}{\mathcal{C}(\omega)}},
$$

where $x_{e}$ is the distance between the apex and the input, expressed in terms of the angle $\theta$ of the cone and the input radius $R$ as $x_{e}=R / \sin (\theta / 2)$.

Noting $\mathcal{D}$ the differentiation operator: $\mathcal{D}(\omega)=i \omega$, the input impedance of a conical bore can be written in the form

$$
\mathcal{S}(\omega)=\frac{\frac{x_{e}}{c} \mathcal{D}(\omega)}{1+\frac{x_{e}}{c} \mathcal{D}(\omega) \mathcal{C}^{-1}(\omega)},
$$

yielding the equivalent scheme in Fig. 7.

As was the case for the cylindrical bore, this scheme shows that the wave variables and the reflection function are embedded in the model and that only the consequence of the propagation is considered.

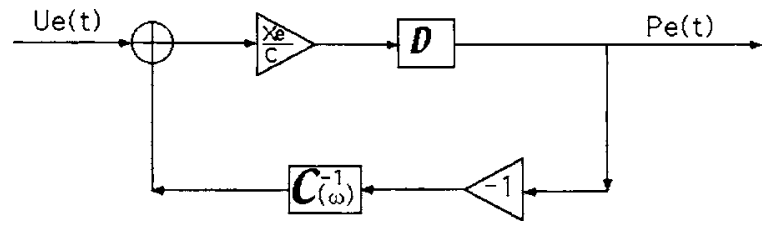

FIG. 7. Impedance model of a conical bore.

Moreover, as opposed to the case of a cylindrical bore, the use of wave variables in the case of a conical bore does not lead to a more compact description of the resonator since the reflection function $\mathcal{R}(\omega)$ is given by

$$
\mathcal{R}(\omega)=-\frac{2 \frac{x_{e}}{c} i \omega \exp (-2 i k(\omega) L)+1-\exp (-2 i k(\omega) L)}{2 \frac{x_{e}}{c} i \omega+1-\exp (-2 i k(\omega) L)} .
$$

Here, $\mathcal{R}(\omega)$ does not reduce to a simple delay+filter and exhibits an instantaneous response proportional to $\Theta(t) \exp \left(-c t /\left(2 x_{e}\right)\right)$ for $t \leqslant 2 L / c$. This is due to the presence of the "air" bore, which is a lumped element. These facts have been largely studied (see, e.g., Ref. 18).

\section{B. Coupling of the resonator with the reed and the nonlinear characteristics}

The system of two coupled equations (9) and (11), combined with any impedance model corresponding to Eq. (10), leads to the introduction of the reed and the nonlinearity as a nonlinear loop linking the output $p_{e}$ to the input $u_{e}$ of the resonator, as shown in Fig. 8 in the case of a cylindrical bore. The output of the model is made of the three coupled variables $p_{e}, u_{e}$, and $x$.

The model is piloted by the length $L$ of the bore, the opening of the reed channel represented by the parameter $\zeta(t)$, and the blowing pressure represented by the parameter $\gamma(t)$.

\section{External pressure}

When a real instrument is simulated, the acoustic pressure in the mouthpiece is not the most perceptively relevant variable and the calculation of the external pressure is necessary. For a cylindrical bore, if we assume that the diameter is small compared to the wavelength of the frequencies

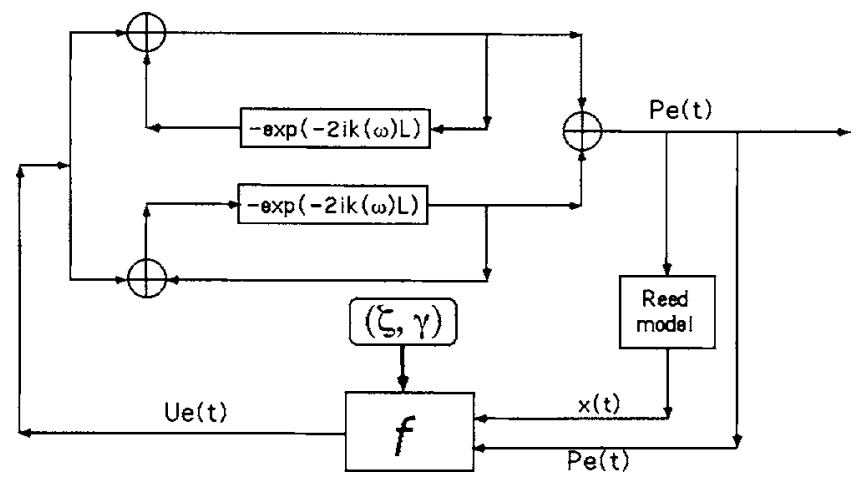

FIG. 8. Synthesis model in the case of a cylindrical bore. 
propagating within the bore, the radiation is monopolar and the external pressure is expressed classically as the time derivative of the output flow: $p_{\text {ext }}(t)=d u_{s}(t) / d t$. Let us express the output flow as a function of the dimensionless variables $p_{e}(t)$ and $u_{e}(t)$. Assuming again that the radiation impedance is negligible $\left[P_{s}(\omega) / U_{s}(\omega)=0\right]$, the following expression is obtained from the transmission line theory:

$$
\begin{aligned}
& P_{e}(\omega)=i \sin (k(\omega) L) U_{s}(\omega), \\
& U_{e}(\omega)=\cos (k(\omega) L) U_{s}(\omega),
\end{aligned}
$$

which yields

$$
U_{s}(\omega)=\exp (-i k(\omega) L)\left(P_{e}(\omega)+U_{e}(\omega)\right) .
$$

From a perceptive point of view, the quantity $\exp (-i k(\omega) L)$ can be ignored, since it represents the delay and the losses encountered by the acoustic pressure during a simple travel between the embouchure and the open end (see Fig. 1). This simplification leads to the following expression of the external pressure:

$$
p_{\text {ext }}(t)=\frac{d}{d t}\left(p_{e}(t)+u_{e}(t)\right) .
$$

This approximation overestimates the high frequencies, but we point out that a deeper physical description of the radiation impedance and radiation losses is beyond the scope of this paper. For a deeper discussion, we refer the reader, e.g., to Ref. 19 for acoustic modeling or Ref. 20 for signal modeling.

\section{DISCRETE-TIME FORMULATION OF THE CONTINUOUS SYSTEM}

In order to draw up the synthesis model, it is necessary to use a discrete formulation in the time domain for the impedance and the reed displacement models.

\section{A. Approximation of the loop filter}

The construction of a discrete version of the impedance relationship first requires an expression of the losses contained in $F(\omega)^{2}=\exp (-2 i k(\omega) L)$ through a digital filter. In order to be modified easily according to the geometry of the resonator, the coefficients of the filter should make an explicit use of the geometrical variables such as the length of the bore and its radius. This requirement led us to express analytically the coefficients of the digital filter as functions of the geometrical parameters, rather than use numerical approximations and minimization techniques to compute the values of these coefficients. For that purpose, we use a classical one pole filter (see, e.g., Ref. 6), written in the following form:

$$
\tilde{F}(\widetilde{\omega})=\frac{b_{0} \exp (-i \widetilde{\omega} D)}{1-a_{1} \exp (-i \widetilde{\omega})},
$$

where $f_{e}$ is the sampling frequency, $\widetilde{\omega}=\omega / f_{e}$, and $D$ $=2 f_{e} L / c$ is the pure delay corresponding to the linear part of $k(\omega)$.
Let us express the parameters $b_{0}$ and $a_{1}$ as functions of the physical parameters, so that $\left|F(\omega)^{2}\right|^{2}=|\tilde{F}(\widetilde{\omega})|^{2}$ for two given values $\omega_{1}$ and $\omega_{2}$ of $\omega$. These two values will be different in the case of a cylindrical bore and in the case of a conical bore and will in both cases correspond to the frequencies of the two first impedance peaks, in order to ensure their exact heights. It is important to preserve this feature for a faithful simulation of the continuous dynamical system, since the linear impedance is coupled with the nonlinear characteristics. It will allow the decay times of the first two modes of the approximated impulse response of the impedance to match those of the exact impulse response, which is important for the transients induced by quick changes of $\gamma(t)$ and $\zeta(t)$. A detailed discussion on the role of the height of the first impedance peaks for the functioning of the clarinet, in particular for the oscillation threshold, can be found, e.g., in Ref. 10.

By ignoring the (small) dispersion introduced by the nonlinear part of the phase of $F(\omega)$ in the cylindrical case, the frequencies of the resonance peaks are given by $\omega_{n}$ $=c \pi(n-1 / 2) / L, n$ being an integer.

In the conical case, the frequencies of the impedance peaks are solutions of the following equation: $\sin (\omega L / c$ $+\pi)=\omega x_{e} / c \cos (\omega L / c)$. In order to express the coefficients of the filter as functions of the physical parameters analytically, we use an approximation of the resonance frequencies, rather than a numerical method to solve this equation. The method consists in finding a first approximation of the solution and use a polynomial expansion around this approximation to find the final approximation. This first approximation is taken as the solution of the equation: $\sin (\omega L / c+\pi)$ $=\cos (\omega L / c)$ and is given by $\omega_{n}=n \pi c / L-\pi c /(4 L)$. Using a first-order limited expansion of $\sin (\omega L / c+\pi)$ and $\omega x_{e} / c \cos (\omega L / c)$ around $\omega=\omega_{n}+\epsilon_{n}$ and identifying the powers of $\epsilon_{n}$ gives directly $\epsilon_{n}=-c\left((4 n-1) x_{e} \pi-4 n L\right) /(L(4 L$ $\left.\left.+(4 n-1) x_{e} \pi+4 x_{e}\right)\right)$. This lets us express the first two frequencies $\omega_{1}$ and $\omega_{2}$ :

$$
\begin{aligned}
& \omega_{1}=\frac{c\left(12 \pi L+9 \pi^{2} x_{e}+16 L\right)}{4 L\left(4 L+3 \pi x_{e}+4 x_{e}\right)}, \\
& \omega_{2}=\frac{c\left(28 \pi L+49 \pi^{2} x_{e}+16 L\right)}{4 L\left(4 L+7 \pi x_{e}+4 x_{e}\right)} .
\end{aligned}
$$

Moreover, for the computation of $\left|F(\omega)^{2}\right|^{2}$ at frequencies $\omega_{1}$ and $\omega_{2}$, the radius $R$ is replaced by an equivalent radius $r_{p}$ defined by $r_{p}=R\left(1+5 L /\left(12 x_{e}\right)\right)$. This value was determined empirically by comparing the impedance of a conical bore obtained by considering the propagation of spherical waves with an impedance of the same conical bore obtained with a concatenation of small elementary cylinders with different diameters (stepped cone), using the transmission line theory.

The system of equations to solve is then given by

$$
\begin{aligned}
& \left|F\left(\omega_{1}\right)^{2}\right|^{2}\left(1+a_{1}^{2}-2 a_{1} \cos \left(\widetilde{\omega}_{1}\right)\right)=b_{0}^{2}, \\
& \left|F\left(\omega_{2}\right)^{2}\right|^{2}\left(1+a_{1}^{2}-2 a_{1} \cos \left(\widetilde{\omega}_{2}\right)\right)=b_{0}^{2},
\end{aligned}
$$

where $\left|F(\omega)^{2}\right|^{2}=\exp (-2 \alpha c \sqrt{\omega / 2} L)$. 

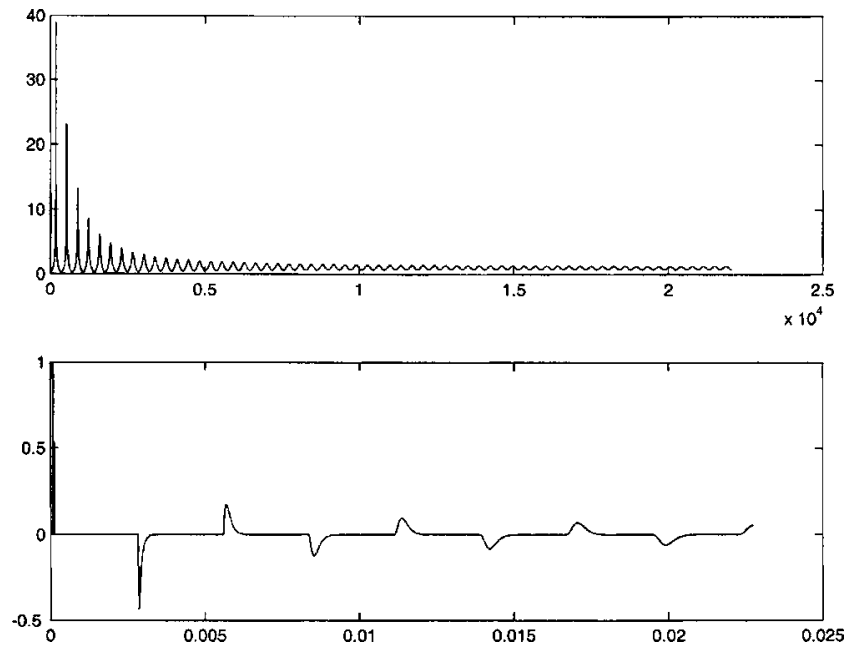

FIG. 9. Top panel: Approximated input impedance ( $f$ in hertz). Bottom panel: Approximated impulse response of the resonator ( $t$ in seconds).

By denoting $c_{1}=\cos \left(\widetilde{\omega}_{1}\right), \quad c_{2}=\cos \left(\widetilde{\omega}_{2}\right), \quad F_{1}=\left|F\left(\omega_{1}\right)^{2}\right|^{2}$, $F_{2}=\left|F\left(\omega_{2}\right)^{2}\right|^{2}, A_{1}=F_{1} c_{1}, A_{2}=F_{2} c_{2}, A_{12}=A_{1}-A_{2}, F_{12}=F_{1}-F_{2}$, the coefficients $a_{1}$ and $b_{0}$ are given by

$$
\begin{aligned}
& a_{1}=\frac{A_{12}-\sqrt{A_{12}^{2}-F_{12}^{2}}}{F_{12}}, \\
& b_{0}=\frac{\sqrt{2 F_{1} F_{2}\left(c_{1}-c_{2}\right)\left(A_{12}-\sqrt{A_{12}^{2}-F_{12}^{2}}\right)}}{F_{12}} .
\end{aligned}
$$

\section{B. Expression of the impedance \\ 1. Cylindrical bore}

From the expression of the input impedance of the cylindrical resonator [Eq. (12)], by denoting $z=\exp (i \widetilde{\omega})$ it becomes directly:

$$
\begin{aligned}
\mathcal{C}(z) & =\frac{1}{1+\left(\frac{b_{0}}{1-a_{1} z^{-1}}\right) z^{-D}}-\frac{\left(\frac{b_{0}}{1-a_{1} z^{-1}}\right) z^{-D}}{1+\left(\frac{b_{0}}{1-a_{1} z^{-1}}\right) z^{-D}} \\
& =\frac{1-a_{1} z^{-1}-b_{0} z^{-D}}{1-a_{1} z^{-1}+b_{0} z^{-D}}
\end{aligned}
$$

yielding the difference equation:

$$
\begin{aligned}
p_{e}(n)= & u_{e}(n)-a_{1} u_{e}(n-1)-b_{0} u_{e}(n-D)+a_{1} p_{e}(n-1) \\
& -b_{0} p_{e}(n-D)
\end{aligned}
$$

Figure 9 shows the approximated input impedance as function of frequency and the impulse response of the resonator computed by the use of the difference equation. The length of the bore is $L=0.5 \mathrm{~m}$ and its radius is $R=7 \mathrm{~mm}$. Comparisons with Fig. 6 show that the heights of the two first impedance peaks are equal, but that the heights of the higher order peaks are smaller. As a consequence, higher order modes decrease faster, yielding an apparently faster decay of the impulse response. Nevertheless, the decays of the first two resonance frequencies are exact. Though this phenomenon might appear as an inconvenience to the use of a first-order low pass filter to model the losses at high frequency, one can also consider it as an advantage. Indeed, it constitutes a way to take into account additional losses in the bore (coming, e.g., from a bell or tone holes radiation) that are not taken into account in the physical model, since they can be ignored at low frequencies but may play a role at higher frequencies.

\section{Conical bore}

In the case of a conical bore, we use the bilinear transformation: $\mathcal{D}(z)=2 f_{e}(z-1) /(z+1)$ to approximate the differentiation operator $\mathcal{D}(\omega)$. The cylindrical bore admittance $C^{-1}(z)$ is given by $C^{-1}(z)=\left(1-a_{1} z^{-1}+b_{0} z^{-D}\right) /\left(1-a_{1} z^{-1}\right.$ $\left.-b_{0} z^{-D}\right)$, and the digital transfer function of the conical bore is

$$
\mathcal{S}(z)=\frac{1}{\frac{z+1}{2 f_{e} \frac{x_{e}}{c}(z-1)}+\frac{1-a_{1} z^{-1}+b_{0} z^{-D}}{1-a_{1} z^{-1}-b_{0} z^{-D}}} .
$$

By denoting $G_{p}=1+c /\left(2 f_{e} x_{e}\right)$ and $G_{m}=1-c /\left(2 f_{e} x_{e}\right)$, the transfer function reduces to

$\mathcal{S}(z)$

$$
=\frac{1-\left(a_{1}+1\right) z^{-1}+a_{1} z^{-2}-b_{0} z^{-D}+b_{0} z^{-D-1}}{G_{p}-\left(a_{1} G_{p}+G_{m}\right) z^{-1}+a_{1} G_{m} z^{-2}+b_{0} G_{m} z^{-D}-b_{0} G_{p} z^{-D-1}}
$$

yielding the difference equation:

$$
\begin{aligned}
p_{e}(n)= & b_{c 0} u_{e}(n)+b_{c 1} u_{e}(n-1)+b_{c 2} u_{e}(n-2) \\
& +b_{c D} u_{e}(n-D)+b_{c D 1} u_{e}(n-D-1) \\
& +a_{c 1} p_{e}(n-1)+a_{c 2} p_{e}(n-2)+a_{c D} p_{e}(n-D) \\
& +a_{c D 1} p_{e}(n-D-1),
\end{aligned}
$$

where the coefficients $b_{c 0}, b_{c 1}, b_{c 2}, b_{c D}$, and $b_{c D 1}$ are defined by

$$
\begin{gathered}
b_{c 0}=\frac{1}{G_{p}}, \quad b_{c 1}=-\frac{a_{1}+1}{G_{p}}, \quad b_{c 2}=\frac{a_{1}}{G_{p}}, \\
b_{c D}=-\frac{b_{0}}{G_{p}}, \quad b_{c D 1}=\frac{b_{0}}{G_{p}}
\end{gathered}
$$

and the coefficients $a_{c 1}, a_{c 2}, a_{c D}$, and $a_{c D 1}$ are defined by

$$
\begin{aligned}
& a_{c 1}=\frac{a_{1} G_{p}+G_{m}}{G_{p}}, \quad a_{c 2}=-\frac{a_{1} G_{m}}{G_{p}}, \\
& a_{c D}=-\frac{b_{0} G_{m}}{G_{p}}, \quad a_{c D 1}=b_{0} .
\end{aligned}
$$

Figure 10 shows the exact and approximated impedance of a conical bore. The characteristics of the bore are: $R$ $=4 \cdot 10^{-3}, L=0.67, \theta=2^{\circ}$. One can notice that the values of the exact and approximated impedances correspond for the frequencies of the first two peaks. 

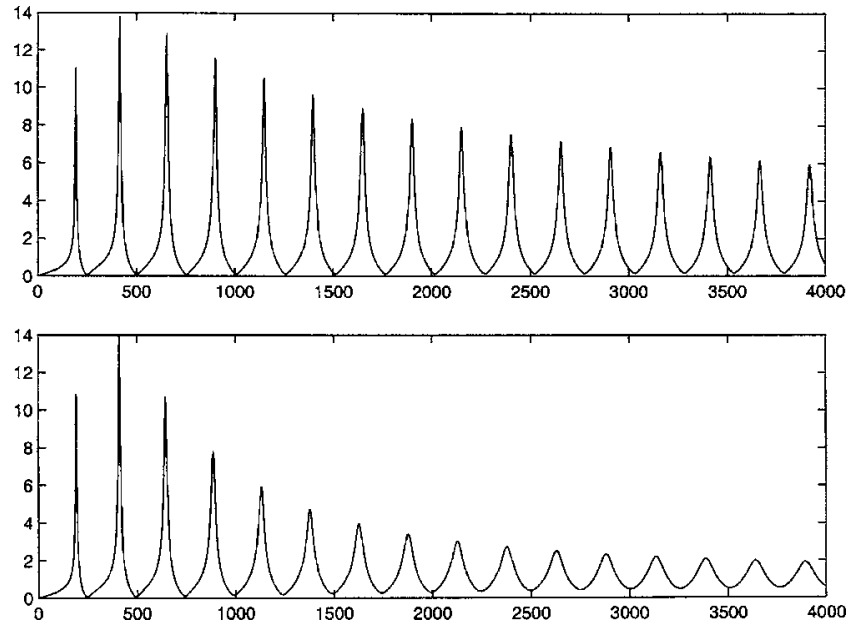

FIG. 10. Top panel: Exact impedance of a conical bore. Bottom panel: Approximated impedance of a conical bore (horizontal axis in hertz).

Figure 11 shows, for the same bore, the impulse response of the exact impedance computed through an inverse Fourier transform, and the impulse response of the approximated impedance computed through the difference equation.

As in the case of the cylindrical bore, the digital approximation of the losses leads to an underestimation of the height of high frequency impedance peaks and a faster decay of the impulse response. Nevertheless, it is worth noticing that the functioning of the whole system (e.g., its oscillation threshold) is mainly driven by the heights and frequencies of the first impedance peaks (see, e.g., Ref. 21 for a justification) and that mainly the nonlinear coupling of the impedance with the exciter, rather than the impedance itself, is responsible of the production of high frequency harmonics.

\section{Approximation of the reed displacement}

Similar to the propagation filter, the relationship between the acoustic pressure and the reed displacement is discretized in the time domain. Since the continuous impulse response of the reed is an exponentially damped sine function satisfying $x(0)=0$, it is natural to build a digital filter in
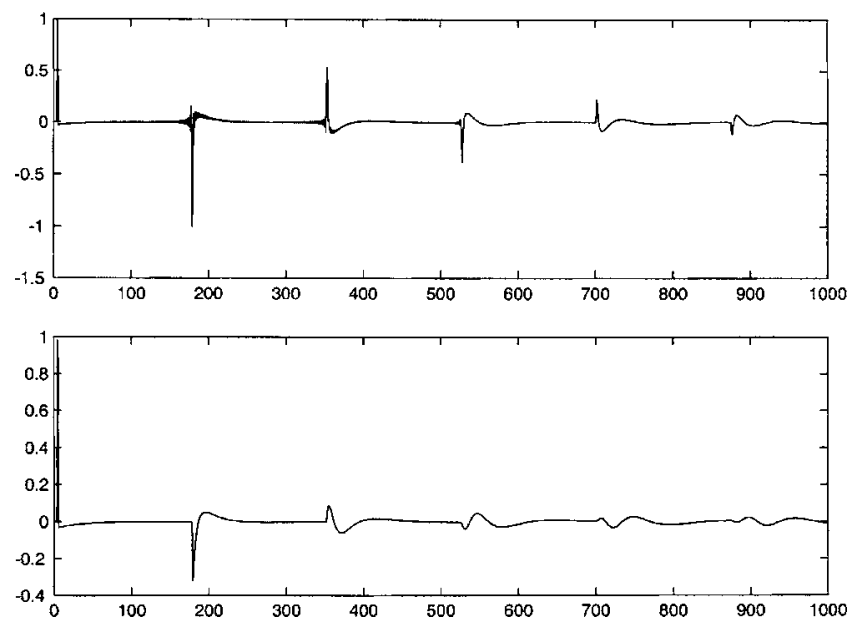

FIG. 11. Top panel: Exact impulse response of a conical bore. Bottom panel: Approximated impulse response of a conical bore (horizontal axis in samples, $\left.f_{e}=44100\right)$. which the displacement of the reed at time $t_{n}=n / f_{e}$ is not a function of the acoustic pressure at time $t_{n}$ but at least at time $t_{n-1}=(n-1) / f_{e}$. It is important to point out that this is not an artificial shift of one sample that may introduce numerical instabilities in the whole scheme, as mentioned, e.g., in Ref. 22 , but that it will let us keep the property $x(0)=0$ of the continuous system when the reed is submitted to a Dirac excitation. In order to satisfy this requirement, rather than using the bilinear transformation or other discretization schemes such as those studied in Ref. 22 to approximate the terms $i \omega$ and $-\omega^{2}$ appearing in the continuous transfer function of the reed, we use $i \omega \simeq f_{e} / 2\left(z-z^{-1}\right)$ and $-\omega^{2} \simeq f_{e}^{2}(z-2$ $\left.+z^{-1}\right)$. These two formulas correspond to classical centered numerical differentiation schemes that are both exact for second-order polynomials. Under these approximations, the digital transfer function of the reed is given by

$$
\begin{aligned}
\frac{X(z)}{P_{e}(z)} & =\frac{\omega_{r}^{2}}{\omega_{r}^{2}+f_{e}^{2}\left(z-2+z^{-1}\right)+\frac{f_{e}}{2}\left(z-z^{-1}\right) q_{r} \omega_{r}} \\
& =\frac{z^{-1}}{\frac{f_{e}^{2}}{\omega_{r}^{2}}+\frac{f_{e} q_{r}}{2 \omega_{r}}-z^{-1}\left(\frac{2 f_{e}^{2}}{\omega_{r}^{2}}-1\right)-z^{-2}\left(\frac{f_{e} q_{r}}{2 \omega_{r}}-\frac{f_{e}^{2}}{\omega_{r}^{2}}\right)}
\end{aligned}
$$

yielding the difference equation:

$$
\begin{aligned}
x(n)= & 0 \times p_{e}(n)+b_{1} p_{e}(n-1)+a_{1} x(n-1) \\
& +a_{2_{a}} x(n-2),
\end{aligned}
$$

where the coefficients $b_{1}, a_{1}$, and $a_{2}$ are defined by

$$
\begin{aligned}
a_{0_{a}}= & \frac{f_{e}^{2}}{\omega_{r}^{2}}+\frac{f_{e} q_{r}}{2 \omega_{r}}, \quad b_{1_{a}}=\frac{1}{a_{0_{a}}}, \quad a_{1_{a}}=\frac{\frac{2 f_{e}^{2}}{\omega_{r}^{2}}-1}{a_{0}}, \\
a_{2_{a}}= & \frac{\frac{f_{e} q_{r}}{2 \omega_{r}}-\frac{f_{e}^{2}}{\omega_{r}^{2}}}{a_{0_{a}}} .
\end{aligned}
$$

Figures 12 and 13 show, respectively, the transfer function and the impulse response of this approximated reed model $\left(f_{r}=2500 \mathrm{~Hz}, q_{r}=0.2\right)$. Comparisons with Figs. 2 and 3 show the relevance of the approximation we used.

\section{Explicit solution of the nonlinear coupled system}

Either in the case of a cylindrical or a conical bore, the difference equation expressing the pressure $p_{e}(n)$ as function of the flow $u_{e}(n)$ can be written in the general form:

$$
p_{e}(n)=b_{c 0} u_{e}(n)+V
$$

where $V=V\left(p_{e}(n-k), u_{e}(n-k)\right), k \geqslant 1$, contains all the terms in Eq. (17) or (18) that are known and do not depend on the time sample $n$. In the case of a cylindrical bore, the value of $b_{c 0}$ is set to $b_{c 0}=1$, according to Eq. (17). Such a notation will let us propose a single resolution scheme valid for any bore geometry. 


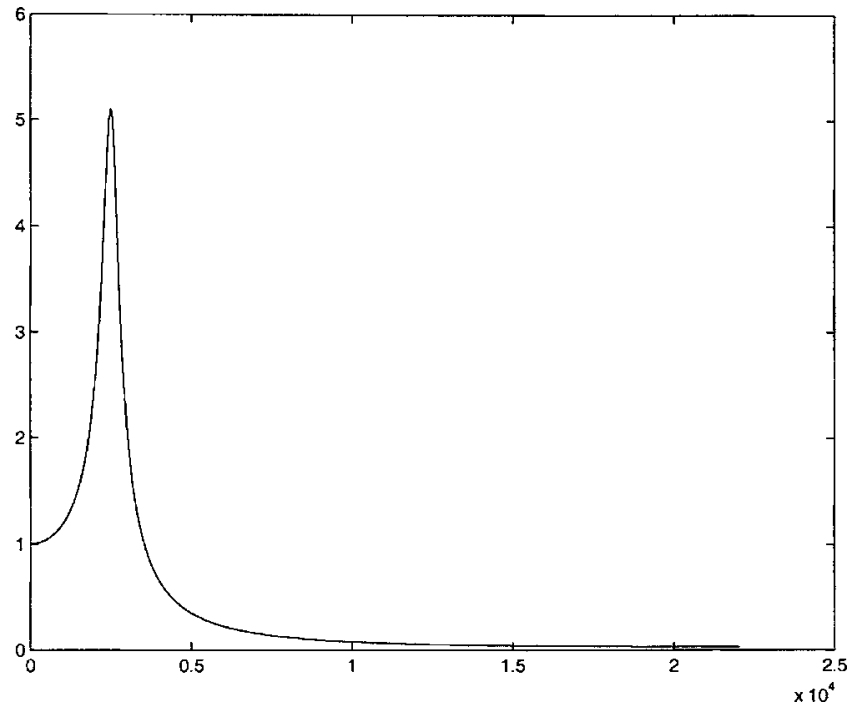

FIG. 12. Transfer function of the approximated reed model (in hertz).

The sampled formulations of the impulse responses of the reed displacement and of the impedance let us write the sampled version of the system of Eqs. (9)-(11):

$$
\begin{aligned}
x(n)= & b_{1_{a}} p_{e}(n-1)+a_{1_{a}} x(n-1)+a_{2} x(n-2), \\
p_{e}(n)= & b_{c 0} u_{e}(n)+V \\
u_{e}(n)= & \Theta(1-\gamma+x(n)) \zeta(1-\gamma+x(n)) \\
& \times \operatorname{sign}\left(\gamma-p_{e}(n)\right) \sqrt{\left|\gamma-p_{e}(n)\right|} .
\end{aligned}
$$

This system of equations is not explicit, but implicit, since the computation of $p_{e}(n)$ with the impedance equation (21) requires the knowledge of $u_{e}(n)$. In a similar way, $u_{e}(n)$ is obtained from the nonlinear equation (22) and requires $p_{e}(n)$.

Such a "delay-free loop" problem could be solved using, e.g., the $\mathbf{K}$-method ${ }^{9}$ for constant values of $\zeta$ and $\gamma$, but thanks to the discretization scheme of the reed displacement, the computation of $x(n)$ with Eq. (20) does not require the

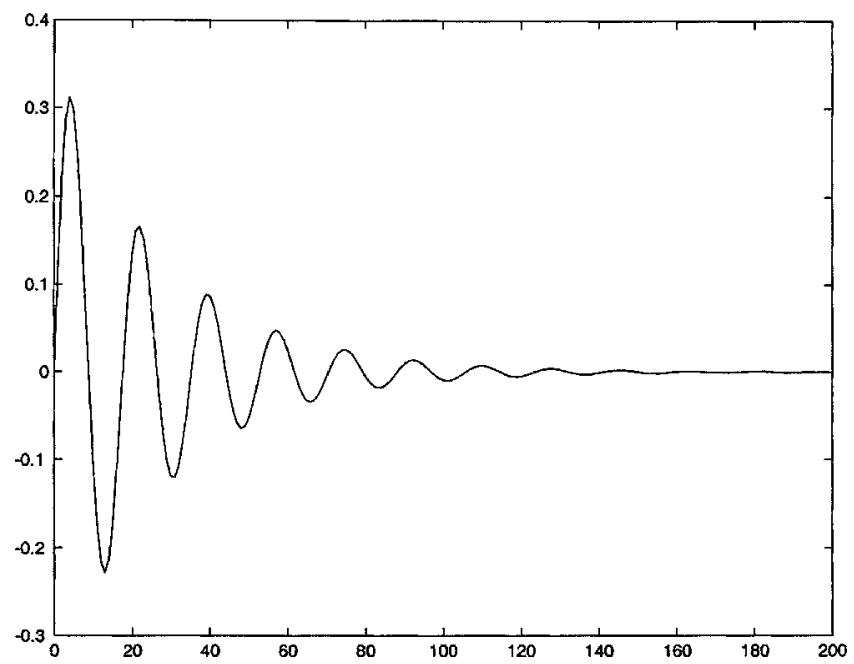

FIG. 13. Impulse response of the approximated reed model (in samples, $\left.f_{e}=44100\right)$. knowledge of $p_{e}(n)$ [but this of $p_{e}(n-1)$ which is known at time $n]$. This makes it possible to solve simply and exactly the coupled system in the case of time-varying values of $\zeta$ and $\gamma$.

For that purpose, we use $W\left[W=W\left(p_{e}(n-k)\right), k \geqslant 1\right]$ to denote all the terms in Eq. (22) that are known at time sample $n$ :

$$
W=\Theta(1-\gamma+x(n)) \zeta(1-\gamma+x(n)) .
$$

With this notation, the two equations (21) and (22) can be written as

$$
\begin{aligned}
& p_{e}(n)=b_{c 0} u_{e}(n)+V, \\
& u_{e}(n)=W \operatorname{sign}\left(\gamma-p_{e}(n)\right) \sqrt{\left|\gamma-p_{e}(n)\right|} .
\end{aligned}
$$

Since the role of the term $\Theta(1-\gamma+x(n))$ is to cancel $W$ when $(1-\gamma+x(n))$ is negative, $W$ always remain positive. We successively consider the two cases: $\gamma-p_{e}(n) \geqslant 0$ and $\gamma$ $-p_{e}(n)<0$ corresponding, respectively, to the cases $u_{e}(n)$ $\geqslant 0$ and $u_{e}(n)<0$.

When $u_{e}(n) \geqslant 0$, substituting the expression of $p_{e}(n)[\mathrm{Eq}$. (24)] into the equation giving $u_{e}(n)[\mathrm{Eq} .(25)]$ gives

$$
u_{e}(n)=W \sqrt{\gamma-\left(b_{c 0} u_{e}(n)+V\right)} .
$$

By taking the square of $u_{e}(n)$ and solving a second-order polynomial in $u_{e}(n)$, this expression yields

$$
u_{e}(n)=\frac{1}{2}\left(-b_{c 0} W^{2} \pm W \sqrt{\left(b_{c 0} W\right)^{2}+4(\gamma-V)}\right) .
$$

In this case, the $(+)$ solution is the only solution for which $u_{e}(n)$ is real and positive $\left[\gamma-V \geqslant 0\right.$ since $\gamma-p_{e}(n) \geqslant 0$ and $\left.b_{c 0} u_{e}(n) \geqslant 0\right]$.

Similarly, when $u_{e}(n)$ is negative, one obtains

$$
u_{e}(n)=-W \sqrt{\left(b_{c 0} u_{e}(n)+V\right)-\gamma},
$$

yielding

$$
u_{e}(n)=\frac{1}{2}\left(b_{c 0} W^{2} \pm W \sqrt{\left(b_{c 0} W\right)^{2}-4(\gamma-V)}\right)
$$

and the $(-)$ solution is the only solution for which $u_{e}(n)$ is real and negative $\left[\gamma-V<0\right.$ since $\gamma-p_{e}(n)<0$ and $b_{c 0} u_{e}(n)$ $<0]$.

From these two solutions, one finally obtains

$$
u_{e}(n)=\frac{1}{2} \operatorname{sign}(\gamma-V)\left(-b_{c 0} W^{2}+W \sqrt{\left(b_{c 0} W\right)^{2}+4|\gamma-V|}\right) .
$$

This solution is unique since the function $\operatorname{sign}(\gamma$ $\left.-p_{e}(n)\right) \sqrt{\left|\gamma-p_{e}(n)\right|}$ is a decreasing monotonous function of $p_{e}$.

The computation of the acoustic pressure and flow in the mouthpiece at time sample $n$ is finally obtained by the sequential use of

$$
\begin{aligned}
& x(n)=b_{1_{a}} p_{e}(n-1)+a_{1_{a}} x(n-1)+a_{2} x(n-2), \\
& V=V\left(p_{e}(n-k), u_{e}(n-k)\right) k \geqslant 1, \\
& W=\Theta(1-\gamma+x(n)) \zeta(1-\gamma+x(n)),
\end{aligned}
$$



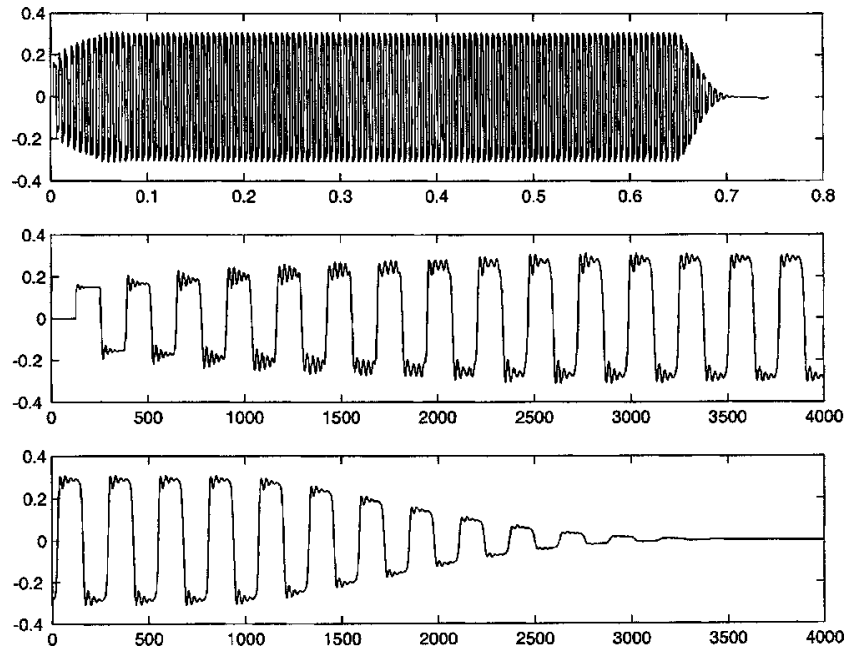

FIG. 14. Top panel: Internal acoustic pressure (in seconds). Middle panel: Blow-up of the attack transient (in samples, $f_{e}=44100$ ). Bottom panel: Blow-up of the decay transient (in samples, $f_{e}=44100$ ).

$$
u_{e}(n)=\frac{1}{2} \operatorname{sign}(\gamma-V)\left(-b_{c 0} W^{2}+W \sqrt{\left(b_{c 0} W\right)^{2}+4|\gamma-V|}\right)
$$

$$
p_{e}(n)=b_{c 0} u_{e}(n)+V .
$$

The calculation of the sampled external pressure $p_{\text {ext }}(n)$ is performed by the use of a difference between the sum of internal pressure and flow at sample $n$ and at sample $n-1$, corresponding to the simplest and classical approximation of the derivative of $p_{\text {ext }}(t)$. This approximation has the advantage of providing a light low-pass filtering compared to the perfect derivation, which "simulates" the radiation losses effects.

It is worth noting that this simple and explicit solution of the discrete nonlinear coupled problem is obtained thanks to the specific discretization scheme of the reed model, which allows a reduction of the complexity of the problem to the solution of a second-order polynomial equation rather than a third-order equation. Though there exist analytic solutions of such an equation, their use is probably not well adapted to a real-time implementation. In the same way, a traveling wave formulation of the whole problem would lead to a similar explicit resolution scheme, provided that the nonlinear characteristics and reed displacement are expressed first with wave variables. In the case of a cylindrical bore, this increase in complexity would be compensated by the simplified formulation of the resonator and the noninstantaneous response of its reflection function, but this gain would no longer remain in the case of a conical bore.

\section{E. Results of simulation}

Figure 14 shows the internal acoustic pressure in the mouthpiece for a cylindrical bore of length $L=0.5$ and radius $R=7 \cdot 10^{-3}$. The values of the parameters are: $\gamma=0.4, \zeta=0.4$, $f_{r}=2205, q_{r}=0.3$. Three phases in the signal are visible: The attack transient corresponding to an abrupt increase of $\gamma$, the
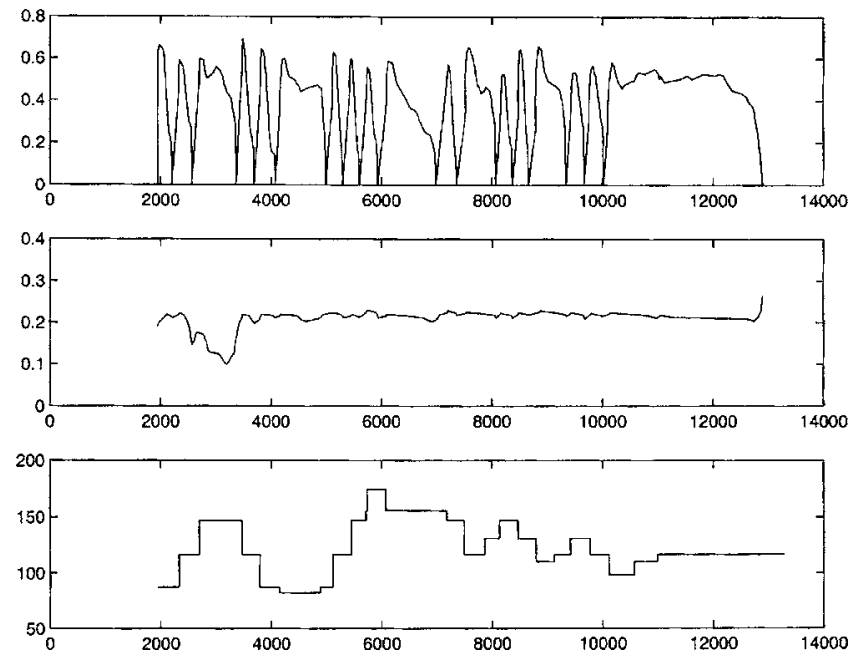

FIG. 15. Top panel: Control parameter $\gamma(t)$. Middle panel: Control parameter $\zeta(t)$. Bottom panel: Frequency of the note (in hertz) (horizontal axis in $\mathrm{ms})$.

steady state oscillations during which $\gamma$ is constant, the decay during which $\gamma$ and $\zeta$ slowly and linearly decrease toward zero.

\section{F. Real-time synthesis implementation}

The synthesis model has been implemented in real-time in the C language as an external Max-MSP object, piloted from MIDI commands given by a Yamaha WX5 controller. This controller measures, as a function of time, the lip pressure controlling the parameter $\zeta(t)$, and the blowing pressure controlling the parameter $\gamma(t)$. This information is received in MIDI format (between 0 and 127) and is scaled so that it corresponds to the range of the physical parameters. The tuning of the model is performed by the use of the MIDI Pitch information coming from the fingering that controls the length $L$ of the bore by the relation $L=c /\left(4 f_{p}\right)$ in the cylindrical case, where $f_{p}$ is the playing frequency. The delay $D$ is implemented through a circular delay line. Like in a real single-reed instrument, since the pitch changes with respect to physical parameters such as $\gamma, \zeta, \omega_{r}$, and $q_{r}$ and since the real instrument is not perfectly tuned for all the fingerings, it seemed unnecessary, at least in a first step, to implement a fractional delay line. The transitions between two notes are handled through a cross-fade in the time domain between the difference equations corresponding to the two different resonator lengths.

In the example to follow, the real-time synthesis model has been used to play the first beats of the "cat" theme of Pierre et le loup by Serge Prokofiev. The sound file can be downloaded at: http://www.lma.cnrs-mrs.fr/ guillemain/ jasa.mp3. The duration of the sequence is $11 \mathrm{~s}$. The reed resonance frequency $\omega_{r} / 2 \pi$ and damping factor $q_{r}$ have been set in order to facilitate the raising of squeaks, respectively, $1850 \mathrm{~Hz}$ and 0.2. During the performance, the external pressure $p_{\text {ext }}$, together with the three playing parameters made of $\zeta(t), \gamma(t)$, and the midi pitch were recorded.

Figure 15 represents, with respect to time (in $\mathrm{ms}$ ), from 


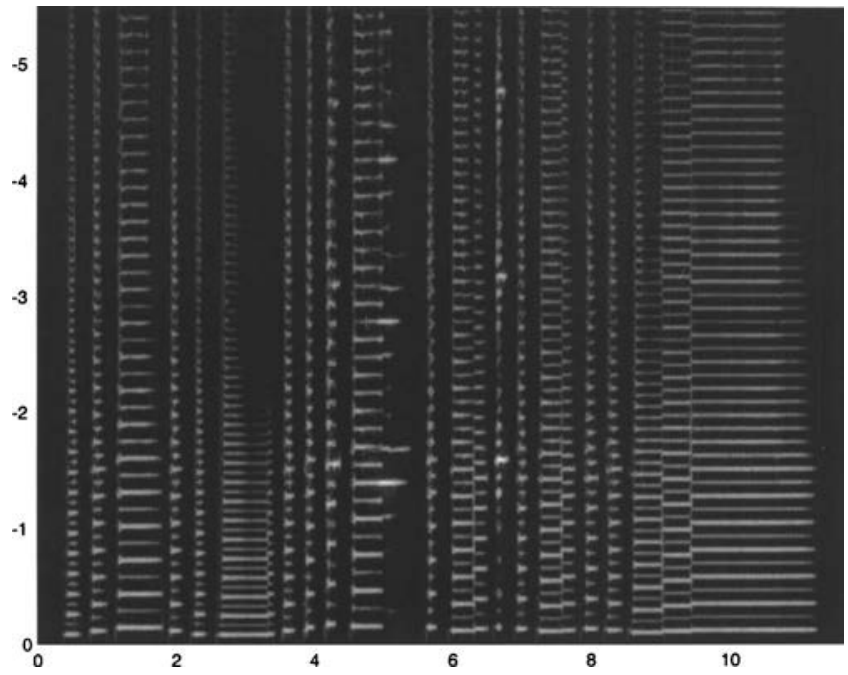

FIG. 16. Spectrogram of the sound sequence (vertical axis in kilohertz, horizontal axis in seconds)

top to bottom, the parameter $\gamma$, the parameter $\zeta$, and the frequency of the midi pitch (in hertz).

Figure 16 shows the spectrogram (between 0 and $5500 \mathrm{~Hz}$ ) of the sound sequence (vertical axis in kilohertz, horizontal axis in seconds).

One can notice that on long sustained notes, the blowing pressure is higher during the attack and decreases when the steady state of the self-oscillations is established. Reed squeaks in the sequence can be seen on the spectrogram around $t=5 \mathrm{~s}$ and $t=6.5 \mathrm{~s}$. In this situation, the reed vibrates at a fundamental frequency located around the impedance peak the frequency of which is close to the reed resonance frequency, producing a treble sound with harmonic content.

Sound examples simulating various wind instruments are available from http://www.lma.cnrs-mrs.fr/ guillemain/ index.html

\section{CONCLUSION}

The real-time synthesis model described in this paper has been obtained through a straightforward transposition of the simplified equations of the physical behavior of a singlereed instrument. In particular, the formulation of the input impedance of the bore, avoiding the classical $P^{+} / P^{-}$decompositions allows the addition of a nonlinear loop modeling the interaction between pressure, reed displacement, and flow to the linear parts of the model, as it is expressed physically. An explicit resolution scheme of the discretized version in time of the coupled system has been proposed. The structure of this scheme makes it possible to extend the model by replacing the linear parts of the system by equations obtained from more complex geometries of the bore and different reed models. Although there are strong connections between this method and other approaches based on wave variables (since the bore is also considered as an acoustic waveguide), working all along the synthesis process with physical variables makes it easy to use refined physical models of the nonlinear characteristics for real-time synthesis ap- plications. Among such refinements, one can mention the additional flow generated by the reed displacement and unsteady terms in the Bernoulli model.

The advantage of this model is its ability to control all the synthesis parameters of the instrument in real-time. Indeed, all the coefficients of the digital filters and the control parameters are explicitly expressed in terms of physical parameters.

Future works using the same approach will present the modeling of other instruments such as saxophones and brasses and the piloting of the synthesis. The vocal tract, for example, could also be modeled through this approach, as has been done, e.g., by Kelly et al. ${ }^{23}$ and Cook $^{24}$ using digital waveguides.

Similarily, mechanical impedance can be modeled using the same formalism. Current works are dealing with the simulation of hammer-string interaction using the nonlinear model described by Hunt et al. ${ }^{25}$

\section{ACKNOWLEDGMENTS}

The authors would like to thank the reviewers for their helpful remarks on the paper, as well as Cécile Le Cocq (presently at ETS, Montréal, Canada).

${ }^{1}$ R. T. Schumacher, "Ab initio calculations of the oscillation of a clarinet," Acustica 48, 71-85 (1981).

${ }^{2}$ B. Gazengel, J. Gilbert, and N. Amir, "Time domain simulation of single reed wind instruments. From the measured input impedance to the synthesis signal. Where are the traps?," Acta Acust. 3, 445-472 (1995).

${ }^{3}$ E. Ducasse, "Modélisation et simulation dans le domaine temporel d'instruments à vent à anche simple en situation de jeu: Méthodes et modèles" (Modeling and simulation in the time domain of single-reed wind instruments in performance situation: Methods and models), Ph.D. thesis, Université du Maine, Le Mans, France, 2001.

${ }^{4}$ J. Gilbert, J. Kergomard, and E. Ngoya, "Calculation of the steady-state oscillations of a clarinet using the harmonic balance technique," J. Acoust. Soc. Am. 86, 35-41 (1989).

${ }^{5}$ J. O. Smith III, "Principles of digital waveguide models of musical instruments," in Applications of DSP to Audio and Acoustics, edited by M. Kahrs and K. Brandenburg (Kluwer, Dordrecht, 1998), pp. 417-466.

${ }^{6} \mathrm{~V}$. Välimäki, "Discrete-time modeling of acoustic tubes using fractional delay filters," Ph.D. Thesis, Helsinki University of Technology, Finland, 1995.

${ }^{7}$ M. van Walstijn and M. Campbell, "Discrete-time modelling of woodwind instrument bores using wave variables," J. Acoust. Soc. Am. 113, 575585 (2003).

${ }^{8} \mathrm{C}$. Vergez, "Trompette et trompettiste: Un système dynamique non linéaire analysé modélisé et simulé dans un contexte musical" (Trumpet and trumpet player: A nonlinear dynamical system analyzed, modelled and simulated in a musical context), Ph.D. thesis, Université Paris 6, France, 2000. ${ }^{9}$ G. Borin, G. De Poli, and D. Rochesso, "Elimination of delay-free loops in discrete-time models of nonlinear acoustic systems," IEEE Trans. Speech Audio Process. 8, 597-606 (2000).

${ }^{10}$ J. Kergomard, S. Ollivier, and J. Gilbert, "Calculation of the spectrum of self-sustained oscillators using a variable truncation method: Application to cylindrical reed instruments," Acust. Acta Acust. 86, 685-703 (2000). ${ }^{11} \mathrm{Ph}$. Guillemain, J. Kergomard, and Th. Voinier, "Procédé de simulation et de synthèse numérique d'un phénomène oscillant" (Process of simulation and digital synthesis of an oscillating phenomenon), French Patent No. FR2846768, Oct 2002, full text available online from http://www.inpi.fr

${ }^{12}$ A. D. Pierce, Acoustics (McGraw-Hill, New York, 1981), presently available from Acoustical Society of America, New York (1990).

${ }^{13}$ J. D. Polack, "Time domain solution of Kirchhoff's equation for sound propagation in viscothermal gases: A diffusion process," J. Acoust. 4, 47-67 (1991).

${ }^{14}$ A. Hirschberg, "Aero-acoustics of wind instruments," in Mechanics of Musical Instruments, edited by A. Hirschberg et al., Lectures Notes CISM (Springer, New York, 1995). 
${ }^{15}$ J. Kergomard, "Elementary considerations on reed-instruments oscillations," in Ref. 14.

${ }^{16}$ T. A. Wilson and G. S. Beavers, "Operating modes of the clarinet," J. Acoust. Soc. Am. 56, 653-658 (1974).

${ }^{17}$ M. R. Schroeder, "Natural sounding artificial reverberation," J. Audio Eng. Soc. 10, 219-233 (1962).

${ }^{18}$ G. Scavone, "An acoustic analysis of single-reed woodwind instruments with an emphasis on design and performance issues and digital waveguide modeling techniques," Ph.D. thesis, Music Department, Stanford University, 1997.

${ }^{19} \mathrm{H}$. Levine and J. Schwinger, "On the radiation of sound from an unflanged circular pipe," Phys. Rev. 73, 383-406 (1948).

${ }^{20} \mathrm{G}$. Scavone, "Modeling wind instrument sound radiation using digital waveguides," Proceedings of the 1999 International Computer Music Con- ference, Beijing, China, pp. 355-358.

${ }^{21}$ A. H. Benade, Fundamentals of Musical Acoustics (Oxford University Press, London, 1976).

${ }^{22}$ F. Avanzini, "Computational issues in physically-based sound models," Ph.D. thesis, University of Padova, Italy, 2001.

${ }^{23}$ L. Kelly Jr. and C. C. Lochbaum, "Speech synthesis," in Proceedings of the Fourth International Congress on Acoustics, Paper G42, Copenhagen, 1962, pp. 1-4.

${ }^{24} \mathrm{P}$. R. Cook, "Identification of control parameters in an articulatory vocal tract model, with applications to the synthesis of singing," Ph.D. thesis, Electrical Engineering Department, Stanford University, 1991.

${ }^{25}$ K. H. Hunt and F. R. E. Crossley, "Coefficient of restitution interpreted as damping in vibroimpact," ASME J. Appl. Mech. 97, 440-445 (1975). 